\title{
Efficient mucosal delivery of optical contrast agents using imidazole-modified chitosan
}

\section{Bilal Ghosn*}

The University of Texas at Austin

Department of Biomedical Engineering

1 University Station, C0800

Austin, Texas 78712

\author{
Anne L. van de Ven* \\ Rice University \\ Department of Bioengineering, MS 142 \\ 6100 Main Street \\ Keck Hall, Suite 116 \\ Houston, Texas 77005 \\ Justina Tam \\ The University of Texas at Austin \\ Department of Biomedical Engineering \\ 1 University Station, C0800 \\ Austin, Texas 78712
}

\section{Ann Gillenwater}

The University of Texas M.D. Anderson Cancer Center Department of Head and Neck Surgery 1515 Holcombe Boulevard

Box 1445

Houston, Texas 77030

\section{Konstantin V. Sokolov}

The University of Texas

M.D. Anderson Cancer Center

Department of Imaging Physics

and

The University of Texas at Austin

1 University Station, C0800

Austin, Texas 78712

\section{Rebecca Richards-Kortum}

Rice University

Department of Bioengineering, MS 142

6100 Main Street

Keck Hall, Suite 116

Houston, Texas 77005

\section{Krishnendu Roy}

The University of Texas at Austin

Department of Biomedical Engineering

1 University Station, C0800

Austin, Texas 78712

\begin{abstract}
The clinical applicability of antibodies and plasmonic nanosensors as topically applied, molecule-specific optical diagnostic agents for noninvasive early detection of cancer and precancer is severely limited by our inability to efficiently deliver macromolecules and nanoparticles through mucosal tissues. We have developed an imidazole-functionalized conjugate of the polysaccharide chitosan (chitosan-IAA) to enhance topical delivery of contrast agents, ranging from small molecules and antibodies to gold nanoparticles up to $44 \mathrm{~nm}$ in average diameter. Contrast agent uptake and localization in freshly resected mucosal tissues was monitored using confocal microscopy. Chitosan-IAA was found to reversibly enhance mucosal permeability in a rapid, reproducible manner, facilitating transepithelial delivery of optical contrast agents. Permeation enhancement occurred through an active process, resulting in the delivery of contrast agents via a paracellular or a combined paracellular/transcellular route depending on size. Coadministration of epidermal growth factor receptor-targeted antibodies with chitosan-IAA facilitated specific labeling and discrimination between paired normal and malignant human oral biopsies. Together, these data suggest that chitosan-IAA is a promising topical permeation enhancer for mucosal delivery of optical contrast agents. (๑) 2010 Society of Photo-Optical Instrumentation Engineers. [DOI: $10.1117 / 1.3309739]$
\end{abstract}

Keywords: epidermal growth factor receptor; early cancer detection; tight junctions; chitosan; tissue permeation; nanoparticle delivery; contrast agent delivery.

Paper 09352R received Aug. 13, 2009; revised manuscript received Dec. 1, 2009; accepted for publication Dec. 8, 2009; published online Feb. 19, 2010.

\section{Introduction}

Early detection is critical for reducing cancer morbidity and mortality. Optical imaging and spectroscopy can be used to interrogate biochemical and architectural changes associated with early epithelial neoplasia to identify suspicious lesions. ${ }^{1}$

*These authors contributed equally to this work.

Address all correspondence to: Krishnendu Roy, The University of Texas at Austin, Department of Biomedical Engineering, 1 University Station, C0800, Austin Texas 78712. Tel: 512-232-3477; Fax: 512-471-0616; E-mail: kroy@mail.utexas.edu.
Molecular-specific optical contrast agents, capable of selectively labeling biomarkers up-regulated during neoplastic progression, have received much interest for their potential to improve early detection. When interrogated optically, these contrast agents can provide dynamic, real-time information about important molecular hallmarks of neoplasia. Studies of small-molecule optical probes with tissue-permeant properties, such as fluorescent sugar derivatives ${ }^{2-6}$ and nucleic acid dyes, ${ }^{7-10}$ have highlighted the promise of using optical imag-

$1083-3668 / 2010 / 15(1) / 015003 / 11 / \$ 25.00$ (C) 2010 SPIE 
ing to distinguish molecular changes in small populations of cells.

In recent years, tremendous progress has been made in developing sophisticated, molecularly targeted contrast agents, including antibodies, quantum dots, and various metal nanosensors (e.g., gold nanoparticles, nanorods, nanoshells, etc.). ${ }^{11-19}$ It has also been demonstrated that targeted gold nanoparticles can provide high contrast between normal and precancerous epithelial tissues. ${ }^{11,20}$ However, their clinical applicability as a topically applied diagnostic agent for early detection of cancer is severely limited, due to our inability to efficiently deliver these agents across mucosal surfaces. Morphological changes associated with precancer generally begin in the basal layers of epithelium; ${ }^{21}$ thus, early-detection strategies require contrast agents to be delivered through several hundred microns of epithelial tissue. It is important that these agents be delivered efficiently and uniformly throughout the region of interest, ensuring that they reach and bind to their targets. In addition, unbound agents should also be easily "washed out" to reduce nonspecific signaling and falsepositive detection. The penetration of molecules through mucosal tissue depends in part on their size and generally decreases exponentially with increasing hydrodynamic volume. ${ }^{22}$ Chemical modification and encapsulation strategies to improve tissue penetration have proven useful only for molecules up to $6 \mathrm{kDa}$ in size. ${ }^{23}$

Permeation enhancers (also called penetration or absorption enhancers) have been investigated to facilitate the delivery of larger molecules. These are topically applied substances that disrupt epithelial tissue by various mechanisms to increase paracellular (between cell) and/or transcellular (across cell) transport. Numerous permeation enhancers have demonstrated efficacy in skin, allowing transdermal penetration of drugs, macromolecules, and nano- and microparticles as large as 1 to $5 \mu \mathrm{m} .{ }^{24-29}$ However, studies utilizing similar formulations in the mucosal epithelium have enhanced the penetration only of small molecules, including insulin $(6 \mathrm{kDa})$, calcitonin $(3.5 \mathrm{kDa})$, and dextrans up to $70 \mathrm{kDa}$ in weight. $^{23,30,31}$ The surfactant Triton-X100 has recently demonstrated reversible mucosal permeation enhancement for efficient delivery of molecules up $150 \mathrm{kDa}$ in size. ${ }^{32,33}$ Nevertheless, the slow rate of tissue recovery associated with Triton$\mathrm{X} 100$ limits the clinical feasibility of this approach, as morphological and histochemical analysis of tissues following treatment with the permeabilizing agent is not highly practical.

Chitosan and its analogs have been studied extensively for in vivo delivery of DNA and siRNA via mucosal delivery routes. ${ }^{34-38}$ Chitosan is a biocompatible, biodegradable, cationic polysaccharide comprised of repeating units of glucosamine and $\mathrm{N}$-acetyl glucosamine. It has been shown to reversibly disrupt epithelial tight junctions to allow the paracellular transport of small molecules. ${ }^{39,40}$ Topical application of chitosan has been shown to enhance drug uptake in nasal, ${ }^{41,42}$ buccal, $^{43,44}$ and intestinal ${ }^{45,46}$ epithelia, and its bioactivity is mediated by its cationic properties. ${ }^{46,47}$ However, a major drawback of chitosan for clinical use has been its limited solubility at physiological $\mathrm{pH}$ and above, which also leads to loss of charge. ${ }^{48,49}$ To improve these characteristics and enhance its permeation properties, we developed a molecular analog in which the primary amines are partially modified with 4-imidazole acetic acid monohydrochloride (henceforth referred to as chitosan-IAA), ${ }^{50}$ thereby introducing secondary and tertiary amines to the polymer structure. We have recently reported that such imidazole-modified chitosan can enhance pDNA expression and siRNA-mediated knockdown in cells when compared to nonmodified chitosan. $^{50}$

In this paper, we describe the first use of chitosan-IAA to deliver tissue-impermeant optical contrast agents through the epithelium of freshly resected mucosal tissues. Topically applied, untargeted macromolecules and nanoparticles were used as probes to assess mucosal permeability following a 15-min pretreatment with chitosan-IAA. Contrast agent localization within the epithelium was interrogated using confocal microscopy to determine the route of transepithelial delivery. The dynamic recovery of barrier function after permeation treatment was assessed using untargeted probes of different sizes. Fluorescently labeled antibodies targeted to epidermal growth factor receptor (EGFR) were delivered into biopsies collected from patients with oral cancer and pretreated with chitosan-IAA to evaluate the clinical potential of this approach. The results of these studies demonstrate that chitosanIAA reversibly enhances mucosal permeability in a rapid, reproducible manner sufficient to facilitate the transepithelial delivery of optical contrast agents up to $44 \mathrm{~nm}$ in diameter.

\section{Materials and Methods}

\subsection{Chemical Modification of Chitosan}

Chitosan was chemically modified to improve its solubility and tissue permeation capacity. The conjugation of 4-imidazole acetic acid monohydrochloride (IAA) to the primary amines of chitosan for production of modified chitosanIAA was performed as previously described. ${ }^{50}$ Briefly, $86 \%$ deacetylated chitosan of $130 \mathrm{kDa}$ molecular weight (PCL 113, Novamatrix, Norway) and IAA (Acros Organics, Morris Plains, New Jersey) were dissolved in $0.1 \mathrm{M}$ 2-( $N$-morpholino)ethanesulfonic acid ( $\mathrm{pH}$ 6.5, Sigma-Aldrich, St. Louis, Missouri) to a concentration of $1.0 \%$ and $2.0 \% \mathrm{w} / \mathrm{v}$, respectively. A theoretical modification of $50 \%$ was targeted for the reaction. IAA and chitosan were combined at a volumetric ratio of 1:5 while on ice. $20 \mathrm{M}$ excess (in relation to IAA) of 1-ethyl-3-(3-dimethylaminopropyl carbodiimide hydrochloride (Pierce Biotechnology, Rockford, Illinois) was added and immediately vortexed to promote addition of IAA to the chitosan backbone. Reactions were allowed to continue overnight with end-over-end mixing. Solutions were dialyzed for 2 days against $5 \mathrm{mM}$ hydrochloric acid (Acros Organics) thrice and then deionized water thrice using Snakeskin pleated dialysis tubing (10,000 MW cut-off; Pierce Biotechnology, Inc.). Following dialysis, the samples were lyophilized for $24 \mathrm{~h}$. Degree of substitution was determined with via ${ }^{1} \mathrm{H}$ NMR. Chitosan and chitosan-IAA were resuspended at a stock concentration of $1 \% \mathrm{w} / \mathrm{v}$ in $0.2 \mathrm{M}$ sodium acetate $(\mathrm{pH}$ 4.5, Sigma Aldrich) immediately prior to dilution for tissue application.

\subsection{Synthesis and Validation of Gold Nanoparticles}

Gold nanoparticles were synthesized as untargeted tissue permeability probes. Gold spheres of $9.5 \pm 0.35 \mathrm{~nm}$ and 
$20.7 \pm 0.26 \mathrm{~nm}$ hydrodynamic diameter were synthesized by a citrate reduction of tetrachloroauric (III) acid $\left(\mathrm{HAuCl}_{4}\right.$, Sigma-Aldrich, St. Louis, Missouri) under reflux, as previously described. ${ }^{51}$ Nanoparticle size was confirmed by dynamic light scattering (DLS) with a ZetaPlus system (Brookhaven Instruments Corporation, Holtsville, New York). Each set of gold nanoparticles were coated with a fluorescent $5 \mathrm{kDa}$ polyethylene glycol (PEG) to reduce their aggregation in media. Briefly, $0.1 \%$ w/v fluorescein-PEG-amine (Laysan Bio, Arab, Alabama) was reacted with $2 \mathrm{M}$ excess of 2-iminothiolane (Pierce Biotechnology) for $2 \mathrm{~h}$. Upon completion, the solution was filtered at room temperature with Amicon Ultra centrifugation filters (3000 MWCO; Millipore, Billerica, Massachusetts) at $3220 \times \mathrm{g}$ for up to $60 \mathrm{~min}$. Gold nanoparticles, suspended at a concentration of 7 $\times 10^{10}$ particles $/ \mathrm{ml}$ (determined by spectral absorbance analysis) were allowed to react with $10^{-4} \mathrm{M}$ fluorescein-PEGthiol at a volumetric ratio of 5:1 for $45 \mathrm{~min}$ on a shaker for each set of nanoparticles. PEG (Sigma-Aldrich) was subsequently added to a final concentration of $2 \% \mathrm{w} / \mathrm{v}$. The particles were centrifuged at $1500 \times \mathrm{g}$ for $30 \mathrm{~min}$ and resuspended in deionized water. Gold coating of nanoparticles was confirmed by monitoring the $\sim 494-\mathrm{nm}$ absorbance peak (absorption maxima of fluorescein on our PEG) using a Synergy HT UV-Vis spectrophotometer (Biotek, Winooski, Vermont). The final hydrodynamic radius of the gold nanoparticles was measured to be $32.9 \pm 1.27 \mathrm{~nm}$ and $43.9 \pm 0.51 \mathrm{~nm}$ using DLS, demonstrating similar size increase following PEGylation as was previously described. ${ }^{52}$

\subsection{Synthesis and Validation of Fluorescent Macromolecules}

Fluorescent macromolecules were prepared as targeted and untargeted tissue permeability probes. These contrast agents were selected based on size, shape, and charge to provide comparison to commonly known and utilized molecular contrast agents. ${ }^{53} 3 \mathrm{kDa}$ rhodamine-dextran, $40 \mathrm{kDa}$ fluoresceindextran, and $150 \mathrm{kDa}$ AlexaFluor 647-IgG were purchased from Invitrogen (Carlsbad, California) for use as untargeted probes. For targeted labeling studies, mouse antihuman antibodies specific for epidermal growth factor receptor (EGFR; clone 108; custom synthesized by the Baylor College of Medicine, Houston, Texas) were reacted with AlexaFluor 647 carboxylic acid succinimidyl esters using commercially available labeling kits (Invitrogen). The purified conjugates were suspended in PBS at a concentration of $1.0 \mathrm{mg} / \mathrm{ml}$. Dyelabeled isotype controls were synthesized at the same concentrations. Prior to tissue labeling, the bioactivity and specificity of the conjugates was confirmed using live EGFR-positive 1483 cells and EGFR-negative MDA-MB-435S cells as described in Ref. 54, in both the presence and absence of chitosan and chitosan-IAA.

\subsection{Topical Permeation of Fresh Bladder Tissue}

Guinea pig bladder mucosa was used as a model tissue to evaluate tissue permeability in the presence and absence of chitosan-based permeation enhancers because of the folding nature of the tissue. The guinea pig bladder allows for effective imaging of both epithelial and stromal layers of the tissue at once. All animals were cared for in accordance with insti- tutional guidelines. The protocols were reviewed and approved by the IACUC at Rice University. This model has been previously validated for the study of topical permeation enhancement. $^{33}$ Whole bladders were excised from 3- to 4-week-old female Hartley guinea pigs (Charles River Laboratories, Wilmington, Massachusetts) directly following animal sacrifice. The bladders were sectioned into 8 to 10 pieces of uniform surface area $(3 \times 3 \mathrm{~mm})$ using a scalpel and washed once in PBS and then immediately used for permeation studies. Two topical permeation enhancers, chitosan and chitosan-IAA, diluted to $0.01 \% \mathrm{w} / \mathrm{v}$ in DMEM/F12 medium (Invitrogen), were evaluated for their ability to increase tissue permeability. Media alone was used as a negative control. $100 \mu \mathrm{L}$ of permeation-enhancing solution was topically applied to the apical surface of each tissue biopsy for $15 \mathrm{~min}$.

\subsection{Assessment of Transepithelial Macromolecule Delivery}

To determine whether chitosan-treated tissues are selectively permeable to macromolecules of specific sizes, fluorescent macromolecules ranging from $3 \mathrm{kDa}$ to $150 \mathrm{kDa}$ in size were topically applied to the tissue surfaces of bladder biopsies. Tissue penetration was then monitored optically. The apical surface of the epithelium was topically treated for $15 \mathrm{~min}$ with prewarmed $\left(37{ }^{\circ} \mathrm{C}\right) 0.01 \% \mathrm{w} / \mathrm{v}$ chitosan or chitosanIAA, washed once in cold media, and covered with a 1:1:1 mixture of $3 \mathrm{kDa}$ rhodamine-dextran, $40 \mathrm{kDa}$ fluoresceindextran, and $150 \mathrm{kDa}$ AlexaFluor 647-IgG, each diluted to a concentration of $1 \mu \mathrm{M}$ in cold media. Tissues were immersed with this solution for $15 \mathrm{~min}$ at $4{ }^{\circ} \mathrm{C}$ and then imaged using confocal microscopy at three different excitation wavelengths (described in the following). Images were collected in $2-$ to $5-\mu \mathrm{m}$ steps from the surface into the tissue. Following imaging, the tissues were washed three times in cold media (15 min total) and reimaged to assess the removal of unbound macromolecules. Experiments were repeated with chitosanIAA treatment at $25^{\circ} \mathrm{C}$ or $4{ }^{\circ} \mathrm{C}$ to determine the influence of temperature on tissue permeation. Each labeling condition was evaluated in six independent experiments.

\subsection{Assessment of Transepithelial Nanoparticle Delivery}

To determine whether chitosan-treated tissues permit the transepithelial delivery of nanoparticles, nanoparticles of different sizes were topically applied to the surface of permeabilized bladder biopsies. Nanoparticle delivery was then monitored optically. Briefly, the apical surface of the epithelium was topically treated for $15 \mathrm{~min}$ with prewarmed $\left(37^{\circ} \mathrm{C}\right) 0.01 \%$ w/v chitosan or chitosan-IAA diluted in DMEM/F12 medium, washed once in cold media, and covered with fluoresceinPEG-gold spheres of 33 or $44 \mathrm{~nm}$ diameter, each diluted to a concentration of $1 \times 10^{6}$ nanoparticles $/ \mathrm{ml}$ in cold media. Tissues were immersed with this solution for $15 \mathrm{~min}$ at $4{ }^{\circ} \mathrm{C}$ and then imaged using confocal microscopy. Images were collected in 2- to 5- $\mu \mathrm{m}$ steps from the surface into the tissue. Following imaging, the tissues were washed three times in cold media (15 min total) and reimaged to assess the removal of unbound nanoparticles. Each labeling condition was evaluated in six independent experiments. 


\subsection{Time-Course Analysis of Tissue Recovery}

To monitor the recovery of bladder epithelial barrier function following the removal of chitosan-IAA, fresh bladder biopsies were treated with $0.01 \% \mathrm{w} / \mathrm{v}$ chitosan-IAA or media for 15 min at $37{ }^{\circ} \mathrm{C}$, washed, and probed with nontargeted contrast agents at regular time intervals during tissue recovery. Briefly, chitosan-IAA and media-treated samples were washed three times with warm or cold media and then allowed to recover in media at $37^{\circ} \mathrm{C}$ or $4{ }^{\circ} \mathrm{C}$. At $0,15,30,60,90$, or 120 min after chitosan-IAA removal, the samples were placed on ice and topically labeled with a 1:1:1 mixture of untargeted fluorescent macromolecules $(3 \mathrm{kDA}$ dextran, $40 \mathrm{kDa}$ dextran, and IgG antibody) or 44-nm gold nanoparticles. Samples were allowed to incubate with the macromolecules or nanoparticles for $15 \mathrm{~min}$ prior to imaging via confocal microscopy. Images were collected $40 \mu \mathrm{m}$ below the tissue surface to allow for optical sectioning of both the epithelium and underlying stroma. Tissue samples were assessed in duplicate using a minimum of three independent experiments.

\subsection{Molecular-Specific Labeling of Human Oral Biopsies}

To demonstrate the feasibility of delivering molecular-specific optical contrast agents targeted against biomarkers of neoplasia, human oral biopsies were co-treated with fluorescent EGFR antibodies and chitosan-IAA. Briefly, paired clinically normal and abnormal biopsies of the oral mucosa were obtained from five consenting patients at the University of Texas M.D. Anderson Cancer Center (MDACC). Patients gave written informed consent, and the clinical protocols were approved by the Institutional Review Boards at MDACC and Rice University. Biopsies were immediately placed and remained in chilled media until they arrived at Rice University. The biopsies were embedded vertically into $3 \% \mathrm{w} / \mathrm{v}$ ultrapure agarose (Invitrogen) to prevent the influx of permeation enhancers and contrast agents at the tissue margins, as previously described. ${ }^{33}$ The apical surface of the epithelium was left free of agarose to facilitate topical labeling. Based on the increased number of epithelial layers in human oral resections, tissues were topically treated with $1 \mu \mathrm{M}$ antiEGFR-647 diluted in $0.01 \% \mathrm{w} / \mathrm{v}$ chitosan-IAA for $60 \mathrm{~min}$ at $37^{\circ} \mathrm{C}$, washed three times with cold media, and then sliced transversely into $200-\mu \mathrm{m}$-thick slices using a Krumdieck tissue slicer. Tissue slices were counterstained with $0.01 \%$ proflavine, a nucleic acid dye, and immediately imaged using confocal microscopy.

\subsection{Confocal Image Acquisition}

All images were obtained using a Carl Zeiss LSM 510 confocal microscope (Thornwood, New York) equipped with 488-nm (30-mW), 543-nm (1-mW), and 633-nm (5-mW) lasers. Images were collected using photo multiplier tube (PMT) detectors and Zeiss LSM 5 image examiner software. Samples were sequentially excited with each laser line with power settings held constant for each laser. Fluorescence emission was collected using 500- to 530-nm (40 kDa fluorescein-dextran), 565- to 615- $\mathrm{nm}$ (3 kDa rhodaminedextran), and 650- to 710-nm (150 kDa Alexa647-IgG) bandpass filters. Tissue reflectance at $633 \mathrm{~nm}$ was collected using a 635-nm dichroic beamsplitter. Images were acquired at 0.5 frames per second using a $20 \times$ objective with a pinhole of 2.56 Airy units. For the untargeted macromolecule and nanoparticle permeation studies, the gain was held constant with excitation at 488, 543, and $633 \mathrm{~nm}$. At the gains utilized, the fluorescence of solutions outside the tissue was oversaturated, but the fluorescence of solutions within the tissue was not. In the EGFR targeting studies, the gain was held constant for antiEGFR-647 imaging (633-nm excitation) and proflavine imaging (488-nm excitation).

\subsection{Image Analysis}

To quantify the fluorescence intensity of the stroma as a function of recovery time and temperature, representative confocal fluorescence images were analyzed using Image J v1.38 (NIH, Bethesda, Maryland). The mean fluorescence intensity per unit area was determined by selecting regions of interest (ROIs) within each image and then dividing the measured fluorescence intensity by the area of the ROI. The ROI margins were drawn just inside the stroma border as determined from reflectance overlay images. The entire stroma was included for each image. When images contained more than one region of discontinuous stroma, the mean intensity for the regions was averaged and treated as a single value. Background noise was determined at each time-point from samples pretreated with media in place of chitosan-IAA. The normalized mean fluorescence intensity of the stroma was calculated by subtracting the measured background and normalizing for the fluorescence intensity of the stroma at time of chitosanIAA removal. The normalized mean fluorescence intensity of the stroma was assessed for each macromolecule using five representative images from three independent experiments (15 images total per macromolecule per time-point and temperature).

The mean reflectance intensity per unit area of the stroma in nanoparticle labeling studies was quantified in the same manner as described for the fluorescence studies, using five representative images from five independent experiments (25 images total per labeling condition).

To quantify the fluorescence intensity of EGFR labeling in transverse images in human oral biopsies, representative confocal fluorescence images were analyzed. In normal samples, ROIs were bounded by the apical and basal surfaces of the epithelium, thereby including any labeling throughout the epithelium. In neoplastic samples, ROIs were drawn to include all squamous cells from the surface of the tissue to the lower margin of EGFR labeling. Regions of fibroblasts, as identified morphologically by proflavine counterstaining, were excluded from the analyses. The mean fluorescence intensity per unit area was assessed using 15 images per biopsy, consisting of three representative images from five separate tissue slices. No background corrections were made because the measured background was $<1 \%$ of the measured mean EGFR labeling intensity for all images evaluated. Differences in mean labeling intensity between normal and neoplastic samples were assessed on a patient-to-patient basis using a two-tailed, unpaired Student's $t$-test, with $p$-values of $\leqslant 0.01$ being considered statistically significant. The relative contrast ratio was determined by dividing the mean fluorescence intensity of each cancer biopsy by that of the contralateral normal. 


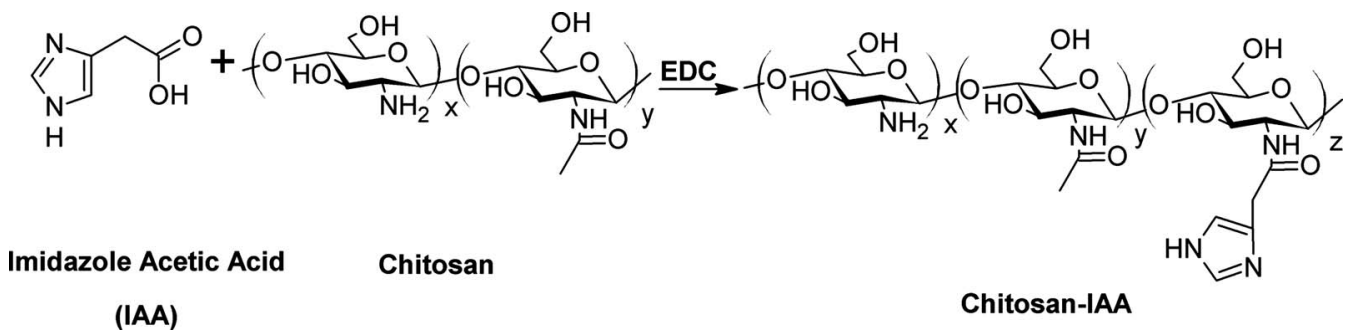

Fig. 1 Chemical schematic for the synthesis of imidazole-modified chitosan (chitosan-IAA).

\section{Results}

\subsection{Synthesis and Characterization of Imidazole- Modified Chitosan}

A schematic for the synthesis of chitosan-IAA is shown in Fig. 1. We successfully introduced imidazole acetic acid to the primary amines of chitosan via a carbodiimide-mediated reaction. Following synthesis, ${ }^{1} \mathrm{H}$ NME characterization of the purified polymer provided on average a degree of substitution of about $3.0 \%$ of the primary amines modified for each chitosan molecule. Chitosan-IAA polysaccharide prepared through this type of reaction has been previously shown to provide enhanced polymer solubility and buffering capacity. ${ }^{50}$

\subsection{Transepithelial Macromolecule Delivery through Fresh Bladder Mucosa}

Figure 2 shows representative confocal fluorescence images of bladder tissue treated topically with $0.01 \% \mathrm{w} / \mathrm{v}$ chitosan-

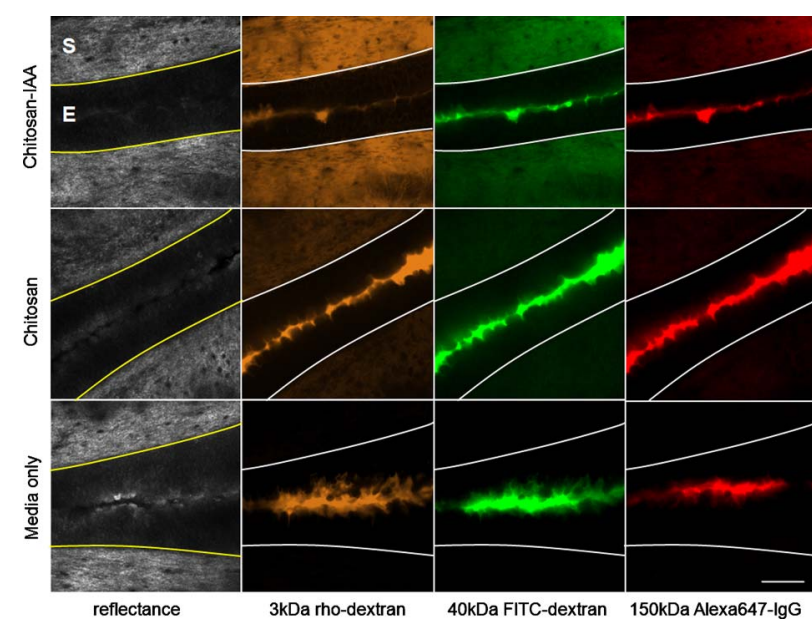

Fig. 2 Stromal accumulation of fluorescent macromolecules following topical permeation treatment. Freshly excised bladder tissue was treated for $15 \mathrm{~min}$ with $0.01 \% \mathrm{w} / \mathrm{v}$ chitosan-IAA, chitosan, or media and then probed with a 1:1:1 mixture of $3 \mathrm{kDa}$ rhodamine-dextran, 40 kDa fluorescein-dextran, and Alexa 647-IgG. The yellow/white lines indicate the boundary between the epithelium and stroma. In reflectance images, the epithelium was distinguished from the stroma by its darker appearance. Following permeation treatment, the fluorescent macromolecules accumulated in the stroma. Stromal accumulation was brighter following treatment with imidazole-modified chitosan than with nonmodified chitosan. In media-treated controls, macromolecule penetration was limited to a few cells in the superficial epithelium. The scale bar represents $100 \mu \mathrm{m}$. The epithelium (E) and stroma (S) are labeled in the first image. (Color online only.)
IAA, chitosan, or media for $15 \mathrm{~min}$ at $37^{\circ} \mathrm{C}$ followed by topical application of a 1:1:1 mixture of $3 \mathrm{kDa}$ rhodaminedextran, $40 \mathrm{kDa}$ fluorescein-dextran, and $150 \mathrm{kDa}$ Alexa647$\mathrm{IgG}$. The tissue reflectance images are shown on the left, and the corresponding fluorescence images are shown to the right. The yellow/white lines indicate the boundary between the stroma and the epithelium. Due to the three-dimensional (3-D) folding of the resected bladder, it was possible to image the epithelium in cross section using confocal microscopy at a depth of $40 \mu \mathrm{m}$. In the reflectance images, the epithelium was distinguished from the stroma by its darker appearance. Both chitosan and chitosan-IAA were found to facilitate the transepithelial delivery of macromolecules. Macromolecules of all three sizes accumulated in the stroma of permeationenhanced tissues, causing the stroma to appear brighter than the epithelium in the fluorescence images. Chitosan-IAA treatment facilitated more intense stromal accumulation than nonmodified chitosan for all sizes of macromolecules evaluated. The macromolecules could be removed with several brief washes in media (data not shown), demonstrating that macromolecule accumulation is reversible. Experiments were independently repeated by labeling tissues with one macromolecule at a time (data not shown) to exclude macromolecule interactions. In media-treated controls, the macromolecule penetration was limited to the superficial epithelium. No significant macromolecule penetration was observed following chitosan-IAA or chitosan treatment at $25^{\circ} \mathrm{C}$ or $4{ }^{\circ} \mathrm{C}$ (data not shown).

\subsection{Transepithelial Nanoparticle Delivery through Fresh Bladder Mucosa}

Figure 3 shows representative confocal fluorescence images of bladder tissue treated topically with $0.01 \%$ w/v chitosanIAA, chitosan, or media for $15 \mathrm{~min} 37^{\circ} \mathrm{C}$ and then probed with fluorescein-PEG-gold spheres of 44 or $33 \mathrm{~nm}$ diameter. Both chitosan and chitosan-IAA were found to facilitate the transepithelial delivery of nanoparticles. Following nanoparticle application, fluorescence was primarily observed in the stroma of permeable tissues. Stromal labeling was generally less uniform than observed with macromolecules. Pretreatment with chitosan-IAA resulted in more intense stromal accumulation than nonmodified chitosan. This difference was more obvious following application of larger nanoparticles. The stromal reflectance was visibly enhanced in samples demonstrating nanoparticle uptake. Tissues exposed to nanoparticles showed a stromal reflectance enhancement of 1.5 to 1.7 (44 nm gold) and 1.7 to 2.1 (33 nm gold) compared to tissues treated with media in place of chitosan following quantifica- 


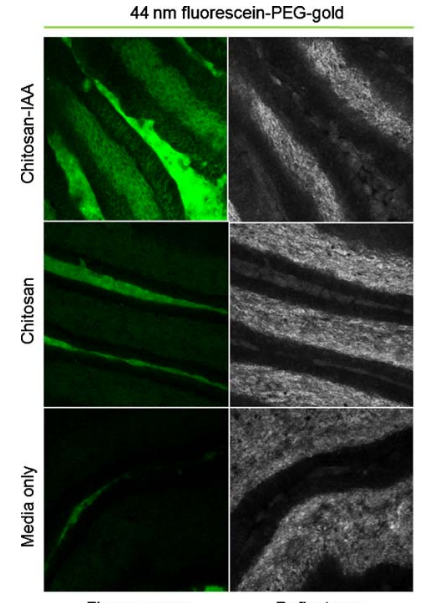

Fluorescence

Reflectance

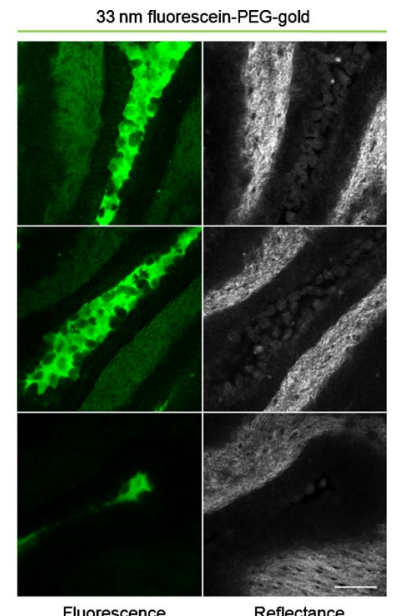

Fluorescence

Reflectance

Fig. 3 Stromal accumulation of gold nanoparticles following topical permeation treatment. Freshly excised bladder tissue was treated for $15 \mathrm{~min}$ with $0.01 \% \mathrm{w} / \mathrm{v}$ chitosan-IAA, chitosan, or media, and then fluorescein-PEG-gold spheres of 44- and 33-nm diameter were applied topically. Transepithelial delivery of both sizes of nanoparticles was observed by monitoring for the localization of nanoparticleassociated fluorescence. The stromal reflectance was visibly enhanced in samples showing accumulation of nanoparticles. No significant change in stromal fluorescence or reflectance was observed in media-treated controls. The scale bar represents $100 \mu \mathrm{m}$.

tion of reflectance at a fixed gain and regions of the stroma. Minimal to no nanoparticle accumulation was observed in media-treated controls.

\subsection{Route of Contrast Agent Delivery Following Chitosan-IAA Treatment}

The route of contrast agent delivery through the epithelium was optically interrogated in fresh bladder tissues pretreated with chitosan-IAA. Confocal fluorescence images were collected in $2-\mu \mathrm{m}$ steps from the surface of the tissue following the topical application of $3 \mathrm{kDa}$ rhodamine-dextran or $44 \mathrm{~nm}$ fluorescein-PEG-gold. Representative videos are available online as Video 1 (3 kDa dextran) and Video 2 (44 nm gold). The videos start at $2 \mu \mathrm{m}$ below the surface and advance at rate of $2 \mu \mathrm{m} / \mathrm{s}$. The stills shown for Video 1 and Video 2 represent frames collected at $10 \mu \mathrm{m}$ below the tissue surface. $3 \mathrm{kDa}$ rhodamine-dextran labeling, shown in Video 1, was characterized by ringlike fluorescence surrounding each cell in the field of view. The labeling appeared extracellular, suggesting a paracellular route of dextran delivery. These rings became progressively smaller in diameter with increasing depth in the epithelium, correlating well with known bladder epithelium morphology. In contrast, the delivery of larger particles, shown in Video 2, appeared to follow both paracellular and transcellular routes. Fluorescein-PEG-gold delivered in a paracellular manner appeared as ringlike labeling that became progressively smaller with increasing depth. Fluorescence associated with nanoparticles was also observed in the cytoplasm of cells across the full thickness of the epithelium, suggestive of transcellular transport. Individual nuclei appeared as a black spot within each cell, suggesting that the transcellular movement of nanoparticles was limited to the cytoplasmic compartment. In both samples, the transition from epithe-

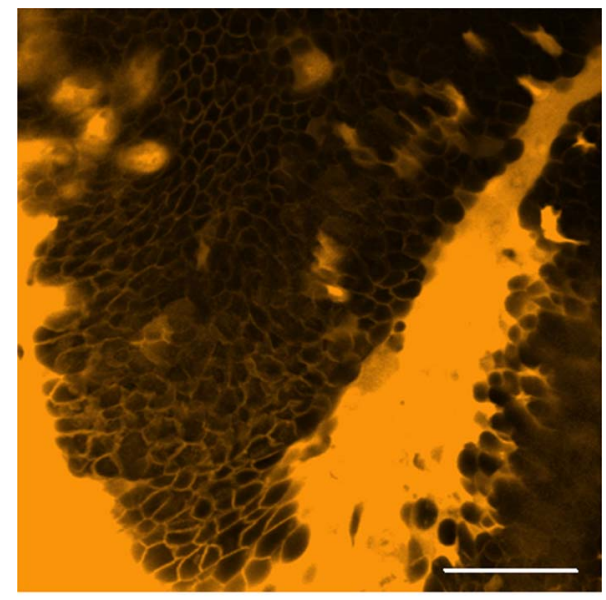

$3 \mathrm{kDa}$ rho-dextran

Video 1 Optical sectioning of bladder epithelium and stroma following the topical application of $3 \mathrm{kDa}$ rhodamine-dextran. The mucosal surface was pretreated with chitosan-IAA30 for 15 min at $37^{\circ} \mathrm{C}$. Confocal fluorescence images were acquired parallel to the tissue surface in $2-\mu \mathrm{m}$ steps with constant laser power and gain. Rhodaminedextran permeation through the epithelium of the tissue appears to follows a paracellular route, appearing as bright rings around cells and minimal labeling within the cells. As the imaging progresses deeper, shown in the video, the stroma appears, clearly defined by a selective accumulation of contrast agents. Representative images, taken at a depth of $10 \mu \mathrm{m}$, are shown here. The scale bar represents $100 \mu \mathrm{m}$ (QuickTime, 3.4 MG).

[URL: http://dx.doi.org/10.1117/1.3309739.1].

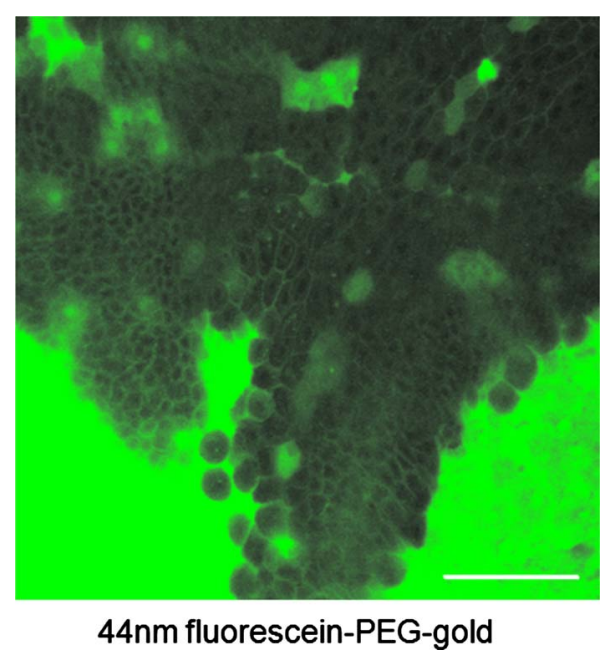

Video 2 Optical sectioning of bladder epithelium and stroma following the topical application of $44 \mathrm{~nm}$ fluorescein-PEG-gold. The mucosal surface was pretreated with chitosan-IAA30 for 15 min at $37^{\circ} \mathrm{C}$. Confocal fluorescence images were acquired parallel to the tissue surface in $2-\mu \mathrm{m}$ steps with constant laser power and gain. Representative images, taken at a depth of $10 \mu \mathrm{m}$, are shown here. Nanoparticle transport in the epithelium appears to follow both paracellular and transcellular routes, appearing as bright rings around cells and diffuse labeling within cells. Individual nuclei appear as a black spot within each cell. Deeper imaging within the tissue samples, seen in the video, demonstrates clear nanoparticle uptake throughout the stromal regions. The scale bar represents $100 \mu \mathrm{m}$ (QuickTime, 3.3 MG). [URL: http://dx.doi.org/10.1117/1.3309739.2]. 


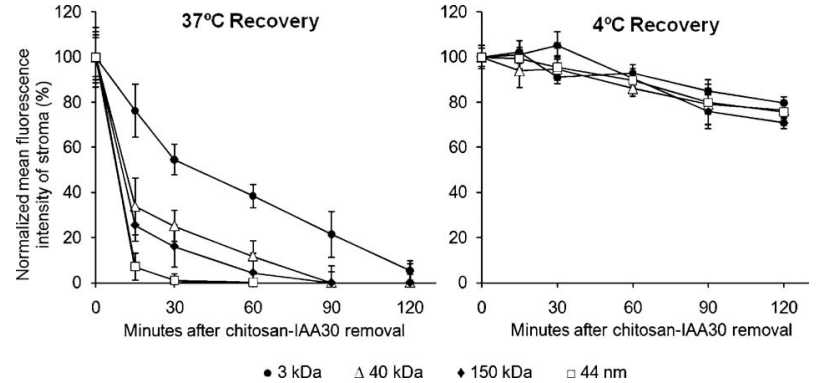

Fig. 4 Fluorescence intensity as a function of time and temperature. Tissues pretreated with chitosan-IAA were washed and allowed to recover at $37^{\circ} \mathrm{C}$ (left) or $4^{\circ} \mathrm{C}$ (right). The recovery of barrier function was probed at regular time intervals using fluorescent macromolecules and nanoparticles. Tissues held at $37^{\circ} \mathrm{C}$ rapidly recovered barrier function, while tissues held at $4{ }^{\circ} \mathrm{C}$ displayed continued permeability as evidenced by stromal fluorescence. The ability of the recovering epithelium to block contrast agents was size dependent, with larger molecules excluded more rapidly than smaller molecules.

lium to stroma (first visible at 14 to $20 \mu \mathrm{m}$ ) was characterized by a relative increase in fluorescence. Dextran and nanoparticle accumulation in the stroma was generally uniform. Few morphological features were defined in stroma, other than blood vessels of horizontal and vertical orientation (see Video 1). These vessels were characterized by lowcontrast agent accumulation, appearing as dark stripes and circles/ovals, respectively. Similar routes of transport for these two contrast agent sizes were seen following pretreatment of tissues with nonmodified chitosan (data not shown).

\subsection{Epithelial Recovery Following Chitosan-IAA Treatment}

Figure 4 shows tissue permeability as a function of time and temperature following the removal of chitosan-IAA. Tissue recovery at $37^{\circ} \mathrm{C}$ is illustrated on the left, and tissue recovery at $4{ }^{\circ} \mathrm{C}$ is illustrated on the right. Samples were pretreated with $0.01 \%$ chitosan-IAA for 15 min and washed in warm or cold media, and then macromolecules or nanoparticles were applied topically at different time-points. The mean fluorescence intensity of the stroma was measured at each time-point to assess epithelial permeability. At $37^{\circ} \mathrm{C}$, the barrier function of the epithelium was found to be recovered quickly, on the order of minutes to hours depending on the size of the permeability probe. The transepithelial delivery of 44-nm nanoparticles was reduced by over $90 \%$ within $15 \mathrm{~min}$ of chitosan-IAA removal. By $90 \mathrm{~min}$, the epithelium remained permeable $(\sim 22 \%$ of initial) only to molecules of $3 \mathrm{kDa}$ in size. With $2 \mathrm{~h}$ of recovery time, the transepithelial delivery of $3 \mathrm{kDa}$ molecules was reduced by $95 \%$. Little tissue recovery was observed for samples held at $4{ }^{\circ} \mathrm{C}$. Macromolecules and nanoparticles of all sizes continued to accumulate in the stroma at all time-points evaluated. The ability of the macromolecules and nanoparticles to reach the stroma was reduced by only $10 \%$ over the course of $2 \mathrm{~h}$. No significant differences were observed between macromolecules or nanoparticles of different sizes at $4{ }^{\circ} \mathrm{C}$. Pretreated tissue that was not washed and that was kept at $37{ }^{\circ} \mathrm{C}$ still retained macromolecule permeation for all sizes for up to $2 \mathrm{~h}$ (data not shown).

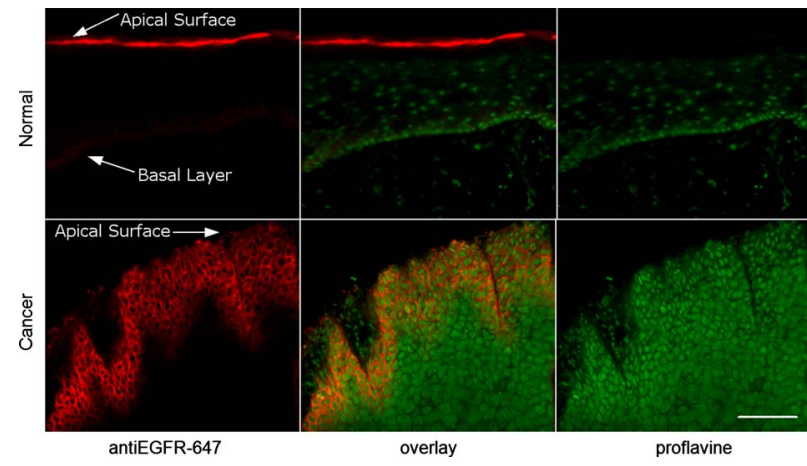

Fig. 5 Representative confocal images of antiEGFR-647 labeling in fresh human oral biopsies co-treated with chitosan-IAA, collected at the same gain. Biopsies were topically treated with antiEGFR-647 diluted in $0.01 \% \mathrm{w} / \mathrm{v}$ chitosan-IAA for $1 \mathrm{~h}$ at $37^{\circ} \mathrm{C}$, washed three times, sliced transversely, and imaged. In normal tissues (top), EGFR labeling is limited to the basal layer of epithelial cells, appearing as a faint region of labeling at the base of the epithelium. Nonspecific labeling is observed at the apical surface of keratinized tissues such as the retromolar trigone tissue shown below. EGFR labeling in squamous cell carcinoma (bottom) is characterized by bright, ring-like extracellular labeling extending downward from the tissue surface. The scale bar represents $100 \mu \mathrm{m}$.

\subsection{Delivery of Targeted Contrast Agents for Cancer Detection}

To demonstrate the clinical potential of chitosan-IAA to aid in delivery of targeted optical contrast agents for the early detection of cancer biomarkers, paired human oral biopsies were topically labeled with fluorescent EGFR-specific antibodies diluted in $0.01 \% \mathrm{w} / \mathrm{v}$ chitosan-IAA. We have previously demonstrated that antibodies targeted to EGFR lack the ability to penetrate normal oral mucosa and squamous carcinomas. ${ }^{33}$ Figure 5 shows representative transverse sections of normal (top) and cancerous (bottom) gingiva tissue labeled for EGFR and counterstained with the nucleic acid dye proflavine. Labeling with antiEGFR-647 is displayed in red and proflavine in green. Differential EGFR labeling was observed between normal and cancer samples from all five patients evaluated. EGFR labeling in normal epithelium was limited to cells in the basal layer. This labeling was observed in all normal samples evaluated, and was generally less intense and more diffuse than labeling in cancer samples. Nonspecific antibody labeling was observed in keratinized tissues, appearing as a bright stripe above the epithelium. EGFR labeling in squamous cell carcinoma tissue was characterized by intense, ringlike labeling extending downward from the tissue surface. This labeling appeared highly uniform, labeling all squamous cells within the treatment zone. Regions of fibroblasts, which were identified by the presence of small, widely spaced nuclei using proflavine staining, did not show significant EGFR labeling. With $1 \mathrm{~h}$ of chitosan-IAA treatment, antibody labeling reached a depth of approximately 150 to $250 \mu \mathrm{m}$ in cancer tissue and $\leqslant 400 \mu \mathrm{m}$ in normal epithelium.

Figure 6 shows the mean fluorescence intensity of paired oral biopsies collected from five patients. The biopsy pairs were labeled and imaged as described earlier. The mean fluorescence intensity of EGFR labeling in normal biopsies was generally low and showed little variation from patient to pa- 


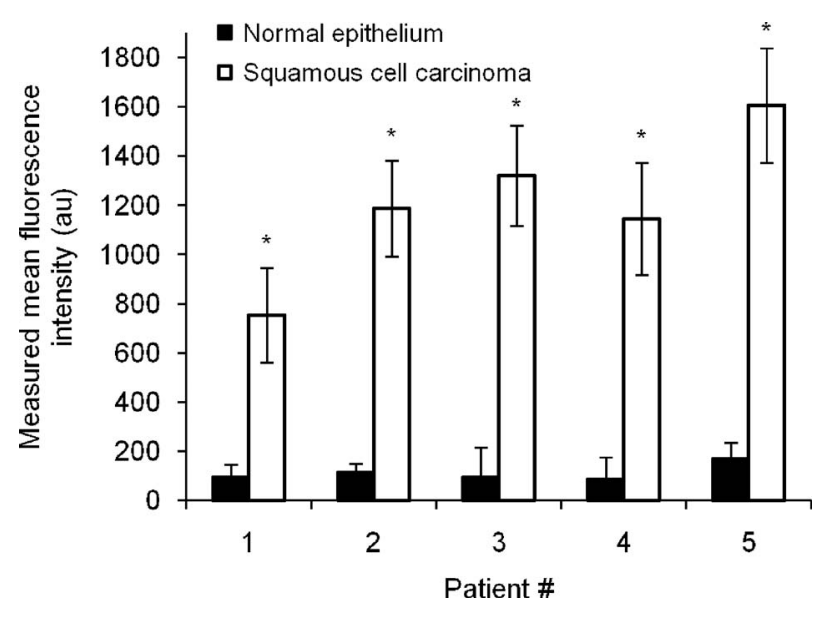

Fig. 6 Measured mean fluorescence intensity of paired oral biopsies collected from patients with cancer of the buccal (1), retromolar trigon (2), maxilla $(3,4)$, and tongue (5) regions. Biopsies were collected from clinically abnormal and contralateral normal regions. The biopsy pairs were topically labeled for EGFR in the presence of chitosan-IAA and imaged transversely. Statistically significant differences $(P \leqslant 0.01)$ were observed in mean fluorescence between biopsy pairs.

tient, despite four different anatomic sites. The intensity of EGFR labeling in squamous cell carcinoma biopsies showed a statistically significant $(p \leqslant 0.01)$ difference when compared to their respective contralateral controls. The relative contrast ratio of cancer-to-normal tissue was found to be 8.1, 10.4, $14.0,13.3$, and 9.5 for patients 1 to 5 , respectively.

\section{Discussion}

Optical interrogation of macromolecule and nanoparticle delivery in freshly resected tissues is a promising approach to study the time-course, route, and efficacy of permeation enhancement. We used confocal microscopy to visualize the localization of targeted and untargeted optical contrast agents following permeation treatment, allowing us to evaluate the potential use of chitosan-IAA to enhance tissue permeability for transepithelial contrast agent delivery. We evaluated molecular contrast agents of various sizes $(3.5 \mathrm{kDa}, 40 \mathrm{kDa}$, $150 \mathrm{kDa}, 33 \mathrm{~nm}$, and $44 \mathrm{~nm}$ ), providing a range representative of potential optical contrast agents with tissueimpermeant properties that are commonly in use. ${ }^{32,33,53}$

The ability of chitosan to enhance the permeation of tissue is highly influenced by the $\mathrm{pH}$ of the environment. ${ }^{46,47}$ Chitosan is insoluble at neutral $\mathrm{pH}$ but is soluble and positively charged at acidic $\mathrm{pH}^{48,49}$ We modified chitosan with 4-imidazole acetic acid monohydrochloride, hypothesizing that the increased solubility and buffering capacity of this molecule would improve transepithelial contrast agent delivery. Results in freshly resected guinea pig bladders show that chitosan-IAA facilitates rapid transepithelial delivery of both macromolecules and nanoparticles. The stromal accumulation of these agents was higher for all sizes evaluated following chitosan-IAA treatment. Efficient delivery was observed following both chitosan-IAA pretreatment and co-treatment, suggesting that complexation with chitosan-IAA is not necessary for enhanced penetration.
The mechanism by which chitosan and its analogs increase tissue permeability remains poorly understood. Experiments using human intestinal Caco-2 cell monolayers have suggested that the mechanism of action, which appears to be mediated by positive charges on the chitosan, ${ }^{46,47}$ includes interactions with tight junction proteins, the redistribution of F-actin, and a destabilization of the cell membrane. ${ }^{55-57}$ Our studies support the observation that this is an active, energydependent process, ${ }^{58}$ since no transepithelial contrast agent delivery was observed following permeation treatment at $25^{\circ} \mathrm{C}$ or $4{ }^{\circ} \mathrm{C}$. Similarly, the recovery of barrier function appears to be an active process, requiring incubation at $37{ }^{\circ} \mathrm{C}$ after washing of the tissue. Permeation of bladder mucosa is rapid, with a 15-min treatment sufficient to facilitate contrast agent transport through several layers of epithelial cells.

The temperature-dependent nature of chitosan-IAA treatment provides a unique opportunity for the study of this process, since the gain and loss of tissue permeability can be halted simply by placing the tissue on ice. Using this approach, we were able to measure size-dependent differences in agent exclusion. Tissues chilled at various time-points during the recovery process preferentially excluded larger contrast agents more rapidly than smaller contrast agents. The ability of the treated tissues to block transepithelial agent delivery increased progressively. Interestingly, in cell monolayers, chitosan concentrations of $\geqslant 0.01 \%$ have been shown to irreversibly block tight junction recovery. In contrast, threedimensional (3-D) tissues washed after treatment with chitosan-IAA show evidence of recovery of the epithelial barrier function. This washing step is important for the rapid reversal of epithelial permeability; however, we have yet to determine whether washing physically removes chitosan-IAA or introduces new divalent cations to compete with chitosanIAA binding sites.

The rapid reversibility of chitosan-IAA mediated permeation supports the clinical potential of this approach. There are concerns that topical permeation enhancers, which by nature interfere with the barrier function of the epithelium, could potentially permit the entry of toxins and/or allergens. Based on our results, we would expect the penetration of these agents to be blocked rapidly. Compared to the topical permeation enhancer Triton-X100 studied in the same tissue model, which has also shown promise for the mucosal delivery of macromolecular optical contrast agents, ${ }^{33}$ tissue recovery following chitosan-IAA treatment is much more rapid. Optimization of the treatment concentration, duration, and removal may be used to further improve the rate of tissue recovery.

The factors that determine the route of contrast agent penetration need to be further clarified. Chitosan solutions have been shown to increase both the paracellular and transcellular permeability of Caco-2 cell monolayers in a reversible, dosedependent manner. ${ }^{59}$ We observed contrast agent uptake in tissues via both these routes. Contrast agents up to $150 \mathrm{kDa}$ in size appeared to follow primarily a paracellular route, while nanoparticles followed both paracellular and transcellular routes. The observed paracellular transport likely resulted from an opening of the tight junctions. The observed transcellular transport was likely a consequence of cell membrane destabilization. Both of these mechanisms have been described in Caco-2 monolayers following chitosan treatment. ${ }^{55-58}$ All contrast agents evaluated here were essen- 
tially neutral in charge (data not shown), suggesting that the shift toward a transcellular route may be determined by probe size. The formulation of chitosan into nanoparticle-drug complexes has been demonstrated to enhance the internalization of chitosan in cells ${ }^{60}$ and tissues, ${ }^{61}$ presenting a second potential approach for driving transcellular delivery. At this time, it is not yet clear whether using chitosan and its analogs as a nanoparticle or nanoparticle coating will be more efficient than chitosan free in solution. From the point of contrast agent delivery, the ability to selectively control the delivery route would be useful for distinguishing between true intracellular labeling and endocytic uptake.

Controlled, uniform delivery of molecular-specific contrast agents across the full thickness of the epithelium remains an important goal for design of early detection strategies. Onetime topical application of $0.01 \%$ chitosan-IAA facilitates the permeation enhancement in a variety of tissues, including the transitional epithelium of the bladder, squamous epithelium of the oral cavity, and squamous cell carcinoma. Delivery of fluorescent EGFR-specific antibodies was observed across the full thickness of the epithelium (150 to $400 \mu \mathrm{m}$ ) in all normal oral biopsies. Differential labeling was observed between normal and cancer specimens, suggesting that the specificity of the antibody and the structure of the target are conserved in the presence of chitosan-IAA. This is in agreement with studies of EGFR labeling in live-cell monolayers in the presence and absence of $0.01 \% \mathrm{w} / \mathrm{v}$ chitosan-IAA (data not shown), where minimal loss of antibody labeling was seen at the equivalent of a $0.01 \% \mathrm{w} / \mathrm{v}$ treatment. The observed differential contrast ratio varies from patient to patient, but EGFR labeling in cancer samples is on average 11 times greater than in normal samples. Experiments to determine the margins of chitosan-IAA treatment will be important for understanding and controlling the 3-D spread of tissue permeation. These margins will need to be distinguished from the labeling margins in order to determine how to further improve the depth of labeling in cancer tissue. The selection of an appropriate animal model will be critical for optimizing the efficacy of chitosan-IAA treatment at doses that can be scaled for clinical use.

The successful translation of chitosan-IAA for in vivo work will require characterizing the safety, toxicity, biological activity, and reversibility of chitosan-IAA mediated permeation at clinically relevant doses. Potential in vivo application would include topical application of chitosan-IAA and contrast agents via gauze pad or similar devices or use of a spray catheter for endoscopically accessible tissues (e.g., gastrointestinal tract and cervix). Chitosan is currently FDA approved as a generally regarded as safe, or GRAS, compound for use as a hemostatic bandage. Drug delivery studies utilizing chitosan derivatives in animal models have demonstrated minimal cytotoxic effects. ${ }^{42,43,62-65}$ Similar studies will be needed to monitor the safety of chitosan-IAA both locally and systemically at regular time intervals following topical treatment. Given the size of the macromolecules and nanoparticles that have been shown to penetrate the tissue following chitosan or chitosan-IAA treatment, study of the potential entry of small viruses should also be analyzed as a potential drawback for in vivo use of the permeation enhancers. Carcinogentreated or orthotopic transplant models will be useful for establishing the sensitivity and specificity of contrast agents de- livered with clinically relevant chitosan-IAA doses. Furthermore, studies for understanding the actual mechanism by which chitosan and chitosan-IAA enhance macromolecular transport are needed. Additional studies are also needed to determine the limitations of chitosan-IAA as a function of concentration, $\mathrm{pH}$, and treatment duration.

\section{Conclusions}

In conclusion, this paper demonstrates that chitosan-IAA is a promising topical permeation enhancer for the mucosal delivery of tissue-impermeant optical contrast agents. Compared to other mucosal permeation enhancers, chitosan and chitosanIAA can facilitate delivery of a broader size range of molecules. No chemical modification of optical contrast agents is needed. Nanoparticles as large as $44 \mathrm{~nm}$ can be delivered following a one-time permeation treatment. Chitosan-IAA, with its improved solubility, appears to be the more effective permeation enhancer, particularly for larger contrast agents. Importantly, permeation of the epithelium can be reversed by washing, leading to a progressive reduction in macromolecule penetration. Recognition that this is an active process provides new avenues for elucidating the mechanism of chitosan and its analogs, since the gain and loss of permeability can be halted simply by placing the treated tissue on ice. The coadministration of chitosan-IAA with molecular-specific contrast agents facilitates differential labeling between normal and cancerous tissues, supporting the clinical potential of this approach. Further work is needed to establish the safety of chitosan-IAA for in vivo use.

\section{Acknowledgments}

We thank Vivian Mack for her assistance with cell culture and animal care. This work was supported in part by NIH BRP Grant No. CA103830.

\section{References}

1. N. Ramanujam, "Fluorescence spectroscopy of neoplastic and nonneoplastic tissues," Neoplasia 2(1-2), 89-117 (2000).

2. N. Nitin, A. L. Carlson, T. Muldoon, A. K. El-Naggar, A. Gillenwater, and R. Richards-Kortum, "Molecular imaging of glucose uptake in oral neoplasia following topical application of fluorescently labeled deoxy-glucose," Int. J. Cancer 124(11), 2634-2642 (2008).

3. J. Levi, Z. Cheng, O. Gheysens, M. Patel, C. Chan, Y. Wang, M. Namavari, and S. Gambhir, "Fluorescent fructose derivatives for imaging breast cancer cells," Bioconjugate Chem. 18(3), 628-634 (2007).

4. K. Yamada, M. Saito, H. Matsuoka, and N. Inagaki, "A real-time method of imaging glucose uptake in single, living mammalian cells," Nat. Protoc. 2(3), 753-762 (2007).

5. R. G. O'Neil, L. Wu, and N. Mullani, "Uptake of a fluorescent deoxyglucose analog (2-NBDG) in tumor cells," Mol. Imaging Biol. 7(6), 388-392 (2005).

6. Z. Cheng, J. Levi, Z. Xiong, O. Gheysens, S. Keren, X. Chen, and S. S. Gambhir, "Near-infrared fluorescent deoxyglucose analogue for tumor optical imaging in cell culture and living mice," Bioconjugate Chem. 17(3), 662-669 (2006).

7. Y. Kakeji, S. Yamaguchi, D. Yoshida, K. Tanoue, M. Ueda, A. Masunari, T. Utsunomiya, M. Imamura, H. Honda, Y. Maehara, and M. Hashizume, "Development and assessment of morphologic criteria for diagnosing gastric cancer using confocal endomicroscopy: an ex vivo and in vivo study," Endoscopy 38(9), 886-890 (2006).

8. T. Muldoon, M. C. Pierce, D. Nida, M. D. Williams, A. Gillenwater, and R. Richards-Kortum, "Subcellular resolution molecular imaging within living tissue by fiber microendoscopy," Opt. Express 15(25), 16413-16423 (2007)

9. T. J. Muldoon, S. Anandasabapathy, D. Maru, and R. Richards- 
Kortum, "High-resolution imaging in Barrett's esophagus: a novel, low-cost endoscopic microscope," Gastrointest. Endosc. 68(4), 737744 (2008).

10. A. L. Polglase, W. J. McLaren, S. A. Skinner, R. Kiesslich, M. F. Neurath, and P. M. Delaney, "A fluorescence confocal endomicroscope for in vivo microscopy of the upper- and the lower-GI tract," Gastrointest. Endosc. 62(5), 686-695 (2005).

11. J. Aaron, N. Nitin, K. Travis, S. Kumar, T. Collier, S. Y. Park, M. Jose-Yacaman, L. Coghlan, M. Follen, R. Richards-Kortum, and K. Sokolov, "Plasmon resonance coupling of metal nanoparticles for molecular imaging of carcinogenesis in vivo," J. Biomed. Opt. 12(3), 034007 (2007).

12. P. Alivisatos, "The use of nanocrystals in biological detection," Nat. Biotechnol. 22(1), 47-52 (2004).

13. J. N. Anker, W. P. Hall, O. Lyandres, N. C. Shah, J. Zhao, and R. P. Van Duyne, "Biosensing with plasmonic nanosensors," Nature Mater. 7(6), 442-453 (2008).

14. N. J. Durr, T. Larson, D. K. Smith, B. A. Korgel, K. Sokolov, and A. Ben-Yakar, "Two-photon luminescence imaging of cancer cells using molecularly targeted gold nanorods," Nano Lett. 7(4), 941-945 (2007).

15. X. Gao, Y. Cui, R. M. Levenson, L. W. Chung, and S. Nie, "In vivo cancer targeting and imaging with semiconductor quantum dots," Nat. Biotechnol. 22(8), 969-976 (2004).

16. X. Huang, I. H. El-Sayed, W. Qian, and M. A. El-Sayed, "Cancer cell imaging and photothermal therapy in the near-infrared region by using gold nanorods," J. Am. Chem. Soc. 128(6), 2115-2120 (2006).

17. K. Sokolov, J. Aaron, B. Hsu, D. Nida, A. Gillenwater, M. Follen, C. MacAulay, K. Adler-Storthz, B. Korgel, M. Descour, R. Pasqualini, W. Arap, W. Lam, and R. Richards-Kortum, "Optical systems for in vivo molecular imaging of cancer," Technol. Cancer Res. Treat. 2(6), 491-504 (2003).

18. J. Yguerabide and E. E. Yguerabide, "Resonance light scattering particles as ultrasensitive labels for detection of analytes in a wide range of applications," J. Cell. Biochem. Suppl 37, 71-81 (2001).

19. M. Stroh, J. P. Zimmer, D. G. Duda, T. S. Levchenko, K. S. Cohen, E B. Brown, D. T. Scadden, V. P. Torchilin, M. G. Bawendi, D. Fukumura, and R. K. Jain, "Quantum dots spectrally distinguish multiple species within the tumor milieu in vivo," Nat. Med. 11(6), 678-682 (2005).

20. K. Sokolov, M. Follen, J. Aaron, I. Pavlova, A. Malpica, R. Lotan, and R. Richards-Kortum, "Gold nanoparticles as molecular specific contrast agents for real time vital optical imaging," Cancer Res. 63(9), 1999-2004 (2003).

21. R. S. Cotran, V. Kumar, and T. Collins, Robbins Pathologic Basis of Disease, W. B. Saunders, Philadelphia (1999).

22. I. Siegel, K. Izutsu, and E. Watson, "Mechanisms of non-electrolyte penetration across dog and rabbit oral mucosa in vitro," Arch. Oral Biol. 26(5), 357-361 (1981).

23. F. Veuillez, Y. N. Kalia, Y. Jacques, J. Deshusses, and P. Buri, "Factors and strategies for improving buccal absorption of peptides," Eur. J. Pharm. Biopharm. 51(2), 93-109 (2001).

24. M. I. Afouna, T. K. Fincher, A. A. A. Zaghloul, and I. K. Reddy, "Effect of azone upon the in vivo antiviral efficacy of cidofovir or acyclovir topical formulations in treatment/prevention of cutaneous HSV-1 infections and its correlation with skin target site free drug concentration in hairless mice," Int. J. Pharm. 253(1-2), 159-168 (2003).

25. H. Gu and K. Roy, "Topical permeation enhancers significantly increase delivery of polymer micro and nanoparticles to epidermal Langerhans' cells," J. Drug Deliv. Sci. Tech. 14(4), 265-273 (2004).

26. K. Takahashi, H. Sakano, N. Numata, S. Kuroda, and N. Mizuno, "Effect of fatty acid diesters on permeation of anti-inflammatory drugs through rat skin," Drug Dev. Ind. Pharm. 28(10), 1285-1294 (2002).

27. D. Southwell and B. W. Barry, "Penetration enhancers for human skin: mode of action of 2-pyrrolidone and dimethylformamide on partition and diffusion of model compounds water, n-alcohols, and caffeine," J. Invest. Dermatol. 80(6), 507-514 (1983).

28. A. C. Williams and B. W. Barry, "The enhancement index concept applied to terpene penetration enhancers for human skin and model lipophilic (estradiol) and hydrophilic (5-fluorouracil) drugs," Int. J. Pharm. 74(2-3), 157-168 (1991).

29. P. P. Sarpotdar and J. L. Zatz, "Percutaneous-absorption enhancement by nonionic surfactants," Drug Dev. Ind. Pharm. 12(11-13), 1625-
1647 (1986).

30. S. Senel, A. J. Hoogstraate, F. Spies, J. C. Verhoef, A. Bos-van Geest, H. E. Junginger, and H. E. Bodde, "Enhancement of in vitro permeability of porcine buccal mucosa by bile salts: kinetic and histological studies," J. Controlled Release 40(1), 45-56 (1994).

31. K. Whitehead, N. Karr, and S. Mitragotri, "Discovery of synergistic permeation enhancers for oral drug delivery," J. Controlled Release 128(2), 128-133 (2008).

32. A. L. van de Ven, K. Adler-Storthz, and R. Richards-Kortum, "Delivery of optical contrast agents using Triton-X100, part 1: reversible permeabilization of live cells for intracellular labeling," J. Biomed. Opt. 14(2), 021012 (2009).

33. A. L. van de Ven, K. Adler-Storthz, and R. Richards-Kortum, "Delivery of optical contrast agents using Triton-X100, part 2: enhanced mucosal permeation for the detection of cancer biomarkers," $J$. Biomed. Opt. 14(2), 021013 (2009).

34. X. Jiang, H. Dai, K. W. Leong, S. H. Goh, H. Q. Mao, and Y. Y. Yang, "Chitosan-g-PEG/DNA complexes deliver gene to the rat liver via intrabiliary and intraportal infusions," J. Gen. Intern Med. 8(4), 477-487 (2006)

35. M. Kumar, A. K. Behera, R. F. Lockey, J. Zhang, G. Bhullar, C. P. De La Cruz, L. C. Chen, K. W. Leong, S. K. Huang, and S. S. Mohapatra, "Intranasal gene transfer by chitosan-DNA nanospheres protects BALB/c mice against acute respiratory syncytial virus infection," Hum. Gene Ther. 13(12), 1415-1425 (2002).

36. J. Pille, H. Li, E. Blot, J. Bertrand, L. Pritchard, P. Opolon, A. Maksimenko, H. Lu, J. Vannier, J. Soria, C. Malvy, and C. Soria, "Intravenous delivery of anti-RhoA small interfering RNA loaded in nanoparticles of chitosan in mice: safety and efficacy in xenografted aggressive breast cancer," Hum. Gene Ther. 17(10), 1019-1026 (2006).

37. F. C. MacLaughlin, R. J. Mumper, J. Wang, J. M. Tagliaferri, I. Gill, M. Hinchcliffe, and A. P. Rolland, "Chitosan and depolymerized chitosan oligomers as condensing carriers for in vivo plasmid delivery," J. Controlled Release 56(1-3), 259-272 (1998).

38. K. Roy, H. Q. Mao, S. K. Huang, and K. W. Leong, "Oral gene delivery with chitosan-DNA nanoparticles generates immunologic protection in a murine model of peanut allergy," Nat. Med. 5(4), 387-391 (1999).

39. M. Hammes and A. Singh, "Effect of polycations on permeability of glomerular epithelial cell monolayers to albumin," J. Lab. Clin. Med. 123(3), 437-446 (1994).

40. A. Kotze, H. Luessen, B. de Leeuw, A. de Boer, J. Verhoef, and H. Junginger, "Comparison of the effect of different chitosan salts and $\mathrm{N}$-trimethyl chitosan chloride on the permeability of intestinal epithelial cells (Caco-2)," J. Controlled Release 51(1), 35-46 (1998).

41. L. Illum, N. F. Farraj, and S. S. Davis, "Chitosan as a novel nasal delivery system for peptide drugs," Pharm. Res. 11(8), 1186-1189 (1994).

42. D. Mei, S. Mao, W. Sun, Y. Wang, and T. Kissel, "Effect of chitosan structure properties and molecular weight on the intranasal absorption of tetramethylpyrazine phosphate in rats," Eur. J. Pharm. Biopharm. 70(3), 874-881 (2008).

43. N. Langoth, H. Kahlbacher, G. Schöffmann, I. Schmerold, M. Schuh, S. Franz, P. Kurka, and A. Bernkop-Schnürch, "Thiolated chitosans: design and in vivo evaluation of a mucoadhesive buccal peptide drug delivery system," Pharm. Res. 23(3), 573-579 (2006).

44. S. Senel, M. J. Kremer, S. Kas, P. W. Wertz, A. A. Hincal, and C. A. Squier, "Enhancing effect of chitosan on peptide drug delivery across buccal mucosa," Biomaterials 21(20), 2067-2071 (2000).

45. A. F. Kotze, H. L. Luessen, B. J. de Leeuw, B. G. de Boer, J. C. Verhoef, and H. E. Junginger, "N-trimethyl chitosan chloride as a potential absorption enhancer across mucosal surfaces: in vitro evaluation in intestinal epithelial cells (Caco-2)," Pharm. Res. 14(9), 1197-1202 (1997)

46. N. G. Schipper, S. Olsson, J. A. Hoogstraate, A. G. deBoer, K. M. Varum, and P. Artursson, "Chitosans as absorption enhancers for poorly absorbable drugs 2: mechanism of absorption enhancement," Pharm. Res. 14(7), 923-929 (1997).

47. H. E. Junginger and J. C. Verhoef, "Macromolecules as safe penetration enhancers for hydrophilic drugs-a fiction?," Pharm. Sci. Technol. Today 1(9), 370-376 (1998).

48. R. Hejazi and M. Amiji, "Chitosan-based gastrointestinal delivery systems," J. Controlled Release 89(2), 151-165 (2003).

49. A. K. Singla and M. Chawla, "Chitosan: some pharmaceutical and 
biological aspects—an update," J. Pharm. Pharmacol. 53(8), 10471067 (2001).

50. B. Ghosn, S. Kasturi, and K. Roy, "Enhancing polysaccharidemediated delivery of nucleic acids through functionalization with secondary and tertiary amines," Curr. Med. Chem. 8(4), 331-340 (2008).

51. G. Frens, "Controlled nucleation for the regulation of the particle size in monodisperse gold suspensions," Nature (London), Phys. Sci. 241, 20-22 (1973).

52. X. Qian, X. H. Peng, D. O. Ansari, Q. Yin-Goen, G. Z. Chen, D. M. Shin, L. Yang, A. N. Young, M. D. Wang, and S. Nie, "In vivo tumor targeting and spectroscopic detection with surface-enhanced Raman nanoparticle tags," Nat. Biotechnol. 26(1), 83-90 (2008).

53. M. C. Pierce, D. J. Javier, and R. Richards-Kortum, "Optical contrast agents and imaging systems for detection and diagnosis of cancer," Int. J. Cancer 123(9), 1979-1990 (2008).

54. E. Hsu, E. Anslyn, S. Dharmawardhane, R. Alizadeh-Naderi, J. Aaron, K. Sokolov, A. El-Naggar, A. Gillenwater, and R. RichardsKortum, "A far-red fluorescent contrast agent to image epidermal growth factor receptor expression," Photochem. Photobiol. 79(3), 272-279 (2004).

55. V. Dodane, M. A. Khan, and J. R. Merwin, "Effect of chitosan on epithelial permeability and structure," Int. J. Pharm. 182(1), 21-32 (1999).

56. N. Fang, V. Chan, H. Q. Mao, and K. W. Leong, "Interactions of phospholipid bilayer with chitosan: effect of molecular weight and pH," Biomacromolecules 2(4), 1161-1168 (2001).

57. M. Thanou, J. C. Verhoef, and H. E. Junginger, "Oral drug absorption enhancement by chitosan and its derivatives," Adv. Drug Delivery Rev. 52(2), 117-126 (2001).
58. G. Ranaldi, I. Marigliano, I. Vespignani, G. Perozzi, and Y. Sambuy, "The effect of chitosan and other polycations on tight junction permeability in the human intestinal Caco-2 cell line," J. Nutr. Biochem. 13(3), 157-167 (2002).

59. N. G. M. Schipper, K. M. Varum, and P. Artursson, "Chitosans as absorption enhancers for poorly absorbable drugs. 1. Influence of molecular weight and degree of acetylation on drug transport across human intestinal epithelial (Caco-2) cells," Pharm. Res. 13(11), 1686-1692 (1996).

60. Z. S. Ma and L. Y. Lim, "Uptake of chitosan and associated insulin in Caco-2 cell monolayers: a comparison between chitosan molecules and chitosan nanoparticles," Pharm. Res. 20(11), 1812-1819 (2003).

61. Z. S. Ma, T. M. Lim, and L. Y. Lim, "Pharmacological activity of peroral chitosan-insulin nanoparticles in diabetic rats," Int. J. Pharm. 293(1-2), 271-280 (2005).

62. S. Dhaliwal, S. Jain, H. Singh, and A. Tiwary, "Mucoadhesive microspheres for gastroretentive delivery of acyclovir: in vitro and in vivo evaluation," AAPS J. 10(2), 322-330 (2008).

63. B. I. Florea, M. Thanou, H. E. Junginger, and G. Borchard, "Enhancement of bronchial octreotide absorption by chitosan and $\mathrm{N}$-trimethyl chitosan shows linear in vitro/in vivo correlation," $J$. Controlled Release 110(2), 353-361 (2006).

64. F. Foger, T. Schmitz, and A. Bernkop-Schnürch, "In vivo evaluation of an oral delivery system for P-gp substrates based on thiolated chitosan," Biomaterials 27(23), 4250-4255 (2006).

65. W. He, X. Guo, and M. Zhang, "Transdermal permeation enhancement of N-trimethyl chitosan for testosterone," Int. J. Pharm. 356(12), 82-87 (2008). 